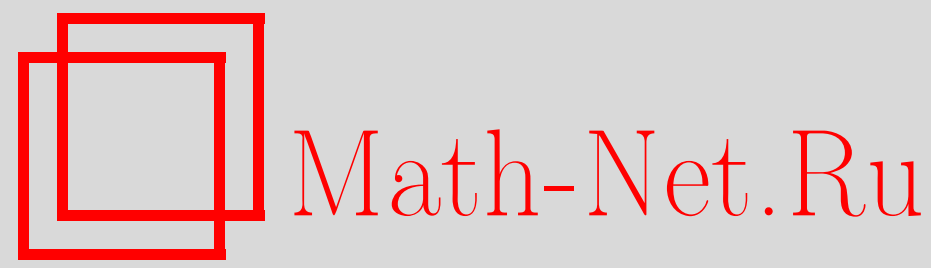

Ф. Н. Гарифьянов, Абсолютно представляющие системы эллиптических функций, Матем. заметки, 1996, том 59, выпуск 6, 932-936

DOI: https://doi.org/10.4213/mzm1794

Использование Общероссийского математического портала MathNet.Ru подразумевает, что вы прочитали и согласны с пользовательским соглашением

http://www . mathnet.ru/rus/agreement

Параметры загрузки:

IP : 34.229 .45 .116

26 апреля 2023 г., 13:31:53

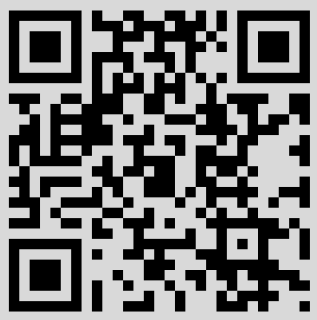




\section{АБСОЛЮТНО ПРЕДСТАВЛЯЮЩИЕ СИСТЕМЫ ЭЛЛИПТИЧЕСКИХ ФУНКЦИЙ}

\section{Ф. Н. Гарифьянов}

В статье изучаются аппроксимативные свойства систем последовательньх производных эллиптических функций. Такие системы являются абсолютно представляющими системами (АПС) в некоторых классах аналитических функций. Далее получены нетривиальные разложения нуля (НPH) по системе производных. Для получения этих результатов предварительно вводятся системы функций, биортогональные к рассматриваемым. Общие вопросы теории $\mathrm{AПC}$ и тесно связанных с ними НРН исследованы в работах Ю.Ф. Коробейника (см., например, [1]). Им же построен и цельй ряд конкретных примеров $\mathrm{AПC}$ целых функций. Рассмотренные в данной статье АПС мероморфных функций имеют некоторые интересные особенности. В частности, естественная область сходимости соответствующих биортогональных рядов, в отличии от хорошо изученного случая АПС целых функций, уже не является ни выпукльм, ни $\rho$-выпуклым множеством. Вместе с тем АПС эллиптических функций и биортогональные к ним системы просты по своей структуре (инвариантность относительно дифференцирования), а каждая эллиптическая функция обладает алгебраической теоремой сложения и периодична (или квазипериодична). Наличие аналогов таких свойств, которьми обладают системы степеней или экспонент, обусловлено относительной простотой эллиптических функций по сравнению с целыми.

Возьмем эллиптическую ю-функцию Вейерштрасса $\wp(z)$, построенную по примитивньм периодам

$$
\omega_{1}=2 \pi, \quad \omega_{2}=2 a ; \quad \operatorname{Im} a>0, \quad|a|=\pi, \quad|a \pm \pi|>\pi,
$$

и рассмотрим четную функцию

$$
f(z)=\wp(z+a+\pi)
$$

которая имеет полюсы в точках

$$
\omega=a+\pi+m_{1} \omega_{1}+m_{2} \omega_{2} .
$$

Пусть $D$ - открытая односвязная область, ограниченная дугами четырех окружностей $|z \pm(a \pm \pi)|=\pi$ и содержащая начало координат. На кусочно-гладкой границе $\partial D$ введем нечетную функцию $\alpha(t)=t \pm(a \pm \pi)$, причем на каждой гладкой компоненте знаки выбраны так, что эта функция взаимно-однозначно отображает $\partial D$ на окружность $E:|z|=\pi$. При этом точки $\pm a, \pm \pi$, которые являются вершинами некоторого прямоугольника $R$, будут для функции $\alpha(t)$ точками разрыва первого рода. В каждой из четырех луночек, вырезаемых областью $D$ из круга $|z| \leqslant \pi$, определим функцию $\alpha(z)$ аналитическим продолжением $\alpha(t)$ с соответствующей гладкой компоненты $\partial D$.

Лемма 1. Системы функиий

$$
\left\{f^{(k)}(z)\right\}, \quad k=0,1, \ldots,
$$

$u$

$$
\left\{(-1)^{m}[(m+1) !]^{-1} \alpha^{m+1}(t)\right\}, \quad m=0,1, \ldots,
$$

биортогональны на 
ДоКАЗАТЕЛЬСТво. Покажем, что

$$
\frac{(-1)^{m}[(m+1) !]^{-1}}{2 \pi i} \int_{\partial D} \alpha^{m+1}(t) f^{(k)}(t) d t=\delta_{m, k}
$$

Из теоремы Коши следует, что интеграл в левой части соотношения (6) можно считать взятым по положительно ориентированной границе прямоугольника $R$. Тогда имеем гомеоморфизм $\alpha(t): \partial R \rightarrow \partial R$ с изменением ориентации, причем $\alpha[\alpha(t)]=t$. Поэтому

$$
\int_{\partial R} \alpha^{m+1}(t) f^{(k)}(t) d t=-\int_{\partial R} t^{m+1} \wp^{(k)}(t) d t
$$

так как $f[\alpha(t)]=\wp(t)$ и последний интеграл вычисляется с помощью вычетов, что и завершает доказательство.

Систему функций (4) пополним 1 , а систему функций (5) - нечетной кусочной постоянной $\beta_{t}$, удовлетворяющей условиям

$$
\int_{\partial D} \beta_{t} d t=2 \pi i, \quad \int_{\partial D} \beta_{t} f(t) d t=0
$$

и имеющей точки разрыва первого рода только в угловых точках $\partial D$. Если на двух соприкасающихся в точке $\pi$ гладких компонентах границы кусочная постоянная $\beta_{t}$ принимает значения $\beta_{1}$ и $\beta_{2}$, то условия (7) равносильны неоднородной системе двух линейных уравнений относительно неизвестных $\beta_{1}$ и $\beta_{2}$. В силу известного соотношения Лежандра [2, с. 176] определитель системы отличен от нуля. Поскольку из-за нечетности и двоякопериодичности

$$
f^{(2 k+1)}( \pm a)=f^{(2 k+1)}( \pm \pi)=0, \quad k=0,1, \ldots
$$

TO

$$
\int_{\partial D} \beta_{t} f^{(k)}(t) d t=0, \quad k=1,2, \ldots
$$

При нечетном $k$ интегралы в левой части (8) обращаются в нуль, как интегралы от четной функции по симметричному относительно начала координат контуру.

Пусть теперь четная функция $\varphi(z)$ голоморфна в $D$ и ее граничное значение $\varphi(t) \in C^{2}(\partial D)$. Класс таких функций обозначим $E_{\varphi}$. Каждой такой функции сопоставим ряд

$$
\varphi(z) \sim \sum_{\nu=0}^{\infty} \mu_{2 \nu} f^{(2 \nu)}(z)+\mu
$$

где

$$
\mu=\frac{1}{2 \pi i} \int_{\partial D} \varphi(t) \beta_{t} d t, \quad \mu_{2 \nu}=\frac{[(2 \nu+1) !]^{-1}}{-2 \pi i} \int_{\partial D} \varphi(t) \alpha^{2 \nu+1}(t) d t
$$

Теорема 1. Ряд (9) сходится $\kappa$ функиии $\varphi(z)$ абсолютно и равномерно по всей замкнутой области $\bar{D}$. 
ДОКАЗАТЕЛЬСТВО. С помощью двукратного интегрирования по частям имеем

$$
2 \pi i(2 \nu+3) ! \mu_{2 \nu}=\left.\alpha^{2 \nu+3}(t) \varphi^{\prime}(t)\right|_{\partial D}-\int_{\partial D} \varphi^{\prime \prime}(t) \alpha^{2 \nu+3}(t) d t
$$

если учесть, что $\alpha^{2 m}(t) \in C(\partial D)$. Поэтому существует такая постоянная $B$, что $\left|\mu_{2 \nu}\right|<B \pi^{2 \nu}[(2 \nu+3) !]^{-1}$. С другой стороны, функция

$$
\theta(z)=f(z)-(z-a-\pi)^{-2}-(z+a+\pi)^{-2}-(z-a+\pi)^{-2}-(z+a-\pi)^{-2}
$$

голоморфна в кругах $|z \pm(a \pm \pi)| \leqslant q$ при некотором $q>\pi$. Но тогда $\mu_{2 \nu} f^{(2 \nu)}(t) \sim$ $[(2 \nu+2)(2 \nu+3)]^{-1}, \nu \rightarrow \infty, t \in \partial D$, т.е. ряд (9) сходится абсолютно и равномерно в области $\bar{D}$. Обозначим через $\varphi_{1}(z)$ разность между суммой ряда и функцией $\varphi(z)$. В силу леммы 1 имеем

$$
\int_{\partial D} \varphi_{1}^{+}(t) \alpha^{m}(t) d t=0, \quad m=\overline{0, \infty},
$$

или, что то же самое,

$$
\int_{|t|=\pi} t^{m} \varphi_{1}^{+}\left[\alpha^{-1}(t)\right] d t=0 .
$$

По теореме Рунге функция $\varphi_{1}^{+}\left[\alpha^{-1}(t)\right]$ является граничным значением функции, голоморфной внутри круга $|z|<\pi$, т.е. она голоморфна и в прямоугольнике $\bar{R} \backslash\{ \pm a, \pm \pi\}$. Вершины прямоугольника могут быть для нее, самое большее, точками разрьва первого рода. Из теории задачи Карлемана со сдвигом $\alpha(t)$ для прямоугольника [3, с. 25] следует, что такое возможно только в одном случае, когда $\varphi_{1}(z)=c, z \in R$. Но должно выполняться и условие $\int_{\partial D} c \beta_{t} d t=0$, т.е. $c=0$, что и завершает доказательство.

ЗАмЕчАниЕ 1. Если снять условие четности, то можно показать, что соответствующий биортогональный ряд сходится к этой функции абсолютно и равномерно на любом компакте $\bar{K} \subset D$.

ЗАмечАниЕ 2. На любом компакте $\bar{K} \subset D$ ряд (9) можно почленно дифференцировать любое число раз как равномерно сходящийся ряд аналитических функций. Если положить, например, $\varphi(z)=z^{2}$, то таким путем получим НРН по системе функций (4).

ЗАМЕчАНИЕ 3 . Если ряд (9) сходится абсолютно хотя бы в одной точке $t_{0} \in \partial D$, то он сходится абсолютно и равномерно во всей области $\bar{D}$.

Теперь рассмотрим канонический оператор Фредгольма

$$
(T \varphi)(t) \equiv \varphi(t)+(A \varphi)(t)
$$

где

$$
(A \varphi)(t) \equiv(2 \pi i)^{-1} g^{+}(t) \int_{\partial D} \varphi(\tau) \psi[\alpha(\tau)-t] d \tau .
$$

Считаем, что $g(z) \in E_{\varphi}$, а нечетная функция $\psi(z)$ голоморфна в круге $|z| \leqslant 2 \pi$. Обозначим через $\widetilde{L}_{2}(\partial D)$ множество четных функций из $L_{2}(\partial D)$. Ясно, что $T: \widetilde{L}_{2}(\partial D) \rightarrow \widetilde{L}_{2}(\partial D)$ и $A: \widetilde{L}_{2}(\partial D) \rightarrow E_{\varphi}$. 
Tеорема 2. Система функиий

$$
1 \cup \beta_{j}(z), \quad \beta_{j}(z)=f^{(2 j)}(z)+g(z) \psi^{(2 j+1)}(z), \quad j=0,1, \ldots,
$$

является АПС в $E_{\varphi}$ тогда и только тогда, когда оператор $T$ обратим.

ДоКАЗАТЕЛЬСТво. Если функция $\gamma(z) \in E_{\varphi}$ и однородное уравнение

$$
(T \varphi)(t)=0
$$

не имеет нетривиальных решений, то уравнение

$$
(T \varphi)(t)=\gamma^{+}(t)
$$

безусловно разрешимо при любой правой части. Его решение $\varphi(t)$ по теореме 1 представимо в $\bar{D}$ равномерно сходящимся рядом (9). Поскольку

$$
\int_{\partial D} f^{(2 j)}(\tau) \psi[\alpha(\tau)-t] d \tau=-\int_{|\tau|=\pi} \wp^{(2 j)}(\tau) \psi(\tau-t) d \tau
$$

то, сосчитав последний интеграл с помощью вычетов, получим разложение функции $\gamma(z)$ по системе функций (14).

Обратно, пусть система функций (14) является АПС в $E_{\varphi}$. Тогда

$$
\gamma^{+}(t)=c_{1}+\sum_{j=0}^{\infty} c_{j} \beta_{j}(t), \quad t \in \partial D,
$$

причем ряд в правой части (17) сходится абсолютно. Но тогда абсолютно и равномерно сходится и ряд

$$
\varphi(t)=c_{1}+\sum_{j=0}^{\infty} c_{j} \beta_{j}(t), \quad t \in \partial D,
$$

который является решением уравнения $(16)$, т.е. оно разрешимо при любой правой части. Решениями союзного уравнения $\left(T^{\prime} \varphi^{*}\right)(t)=0$ будут функции $\varphi^{*}[\alpha(t)]$, где $\varphi^{*}(z)$ - нечетная функция, аналитическая в круге $|z| \leqslant \pi$. Если условие разрешимости

$$
\int_{\partial D} \gamma^{+}(t) \varphi^{*}[\alpha(t)] d t=0
$$

выполняется для любой функции $\gamma^{+}(t)$, то $\varphi^{*}[\alpha(t)]=0$, что и завершает доказательство.

ЗАмечание 4 . Если оператор $T$ не является обратимьм, то система функций (14) будет АПС, если только пополнить ее, самое большее, конечным числом функций. В качестве таких функций можно взять, например, степени $\left\{z^{2 n_{k}}\right\}, k=\overline{1, m}$. Степени $n_{k}$ выбираются так, чтобы уравнение

$$
(T \varphi)(t)=\gamma^{+}(t)+\sum_{k=1}^{m} \alpha_{k} z^{2 n_{k}}
$$

было разрешимо при любой функции $\gamma(z)$ за счет подбора постоянных $\alpha_{k}$. 
ЗАмЕчАнИЕ 5. В некоторых частных случаях условия обратимости оператора $T$ можно найти в явном виде. Пусть, например, $g(z)=\lambda$, а функция $\psi(z)$ голоморффна и $2 \pi$-периодична в полосе $|\operatorname{Im} z| \leqslant 2 \pi$. Тогда

$$
\psi[\alpha(\tau)-t]= \begin{cases}\psi(\tau-a+\pi-t), & \operatorname{Im} \tau>0 \\ \psi(\tau+a+\pi-t), & \operatorname{Im} \tau<0\end{cases}
$$

и с помошью теоремы Коши уравнение (15) запишется как

$$
\varphi(t)+\lambda \int_{-\pi}^{\pi} \varphi[\psi(\tau+a+\pi-t)-\psi(\tau-a+\pi-t)] d \tau=\gamma(t) .
$$

Это уравнение типа свертки с $2 \pi$-периодическим ядром, которое исследуется в замкнутой форме [4, с. 233].

Обсудим теперь возможные обобщения на случай произвольной эллиптической функции $f(z)$, имеющей полюса только в точках (3) и удовлетворяющей условиям

$$
f(z+2 \pi)=f(z) \exp \left(\gamma_{1}\right), \quad f(z+2 a)=f(z) \exp \left(\gamma_{2}\right) .
$$

Пусть сначала

$$
\gamma_{1} \omega_{2}-\gamma_{2} \omega_{1} \neq 2 \pi i\left(n_{1} \omega_{1}+n_{2} \omega_{2}\right),
$$

где $n_{1}$ и $n_{2}$ - фиксированная пара целых чисел. Тогда существует эллиптическая функция порядка 1 , и биортогональной к системе ее производных (4) будет система функций

$$
\left\{A_{m} \theta_{t} \alpha^{m}(t)\right\}, \quad m=0,1, \ldots .
$$

Здесь кусочная постоянная $\theta_{t}$ определяется из равенства $\theta_{\alpha(t)} f[\alpha(t)]=f(t-a-\pi)$, a $A_{m}$ - некоторые постоянные. Теорема 1 остается справедливой, если в определении класса $E_{\varphi}$ условие четности заменить условием

$$
\theta_{\alpha^{-1}(t)} \varphi\left[\alpha^{-1}(t)\right] \in C(|t|=\pi),
$$

которое обеспечивает абсолютную и равномерную сходимость ряда всюду в замыкании $\bar{D}$. При этом нет надобности в пополнении систем (4) и (19).

Если же в (18) имеет место знак равенства, то наименьший возможный порядок функции $f(z)$ равен 2 , т.е.

$$
f(z)=\exp (d z) \wp(z+a+\pi)
$$

при некотором выборе постоянной $d$. Система функций (19), из которой исключена кусочная постоянная, и система производных (4) будут биортогональны только при условии $\operatorname{res}_{z=a+\pi} f(z)=0 \Rightarrow d=0$. Именно этот случай и был подробно изучен в статье.

Казанский авиационный институт

\section{СПИСОК ЦИТИРОВАННОЙ ЛИТЕРАТУРЫ}

1. Коробейник Ю.Ф. // Изв. вузов. Матем. 1992. № 7. С. 26-35. 2. Гурвиц А., Курант Р. Теория функций. М.: Наука, 1968. 3. Чибрикова Л. И. // Уч. записки Казанск. ун-та. 1963. Т. 123. №9. С. 15-39. 4. Гахов Ф. Д., Черский Ю. И. Уравнения типа свертки. М.: Наука, 1978. 